\title{
Scientists set out to connect the dots on long COVID
}

\author{
With grit, urgency and creativity, scientists address the puzzle of long-haul COVID.
}

\author{
Vivien Marx
}

$\mathrm{T}$ oo many have died from COVID-19, but fortunately many have recovered, most without the need for hospitalization. Yet many recoverees are plagued by often life-derailing symptoms such as breathing problems, deep fatigue, joint pain, 'brain fog' and heart palpitations. Long COVID will affect, and already is affecting, millions of people and needs to be taken seriously, says Adrian Hayday, an immunologist at the Francis Crick Institute. Data are still emerging, says Karolinska Institute researcher Petter Brodin, but to a first approximation it appears that $70-80 \%$ of people experiencing severe acute reactions to COVID-19 are men, whereas women comprise $70-80 \%$ of those suffering from long COVID. The average age of long-haul patients is 40 , says neuroimmunologist Avi Nath, who is intramural clinical director of the National Institute for Neurological Disorders and Stroke (NINDS) at the US National Institutes of Health (NIH). "They are in the most productive phases of their lives."

Long COVID may very well have multiple causes, say Hayday and Brodin. Yale University immunologist Akiko Iwasaki and others have also noted that long COVID is likely to involve multiple types of conditions with different causes ${ }^{1,2}$. For example, autoantibodies could play a part, with the immune system attacking the body as it does in rheumatoid arthritis ${ }^{3}$. Perhaps viral reservoirs or lingering fragments of viral RNA or proteins contribute to the condition. The exact cause of long-COVID is currently unknown, says Rockefeller University researcher Jean-Laurent Casanova, who also has a lab at Necker Hospital in Paris. Viral diseases have long been studied, but COVID-19 is a "new disease" that pushes the research community and the world more generally into "uncharted territories," says Casanova.

Deep sleuthing is getting underway to explore scientists' hunches on the underpinnings of long-haul COVID. Resources are being set up to explore how to alleviate symptoms. The UK National Health Service, for example, has set up a

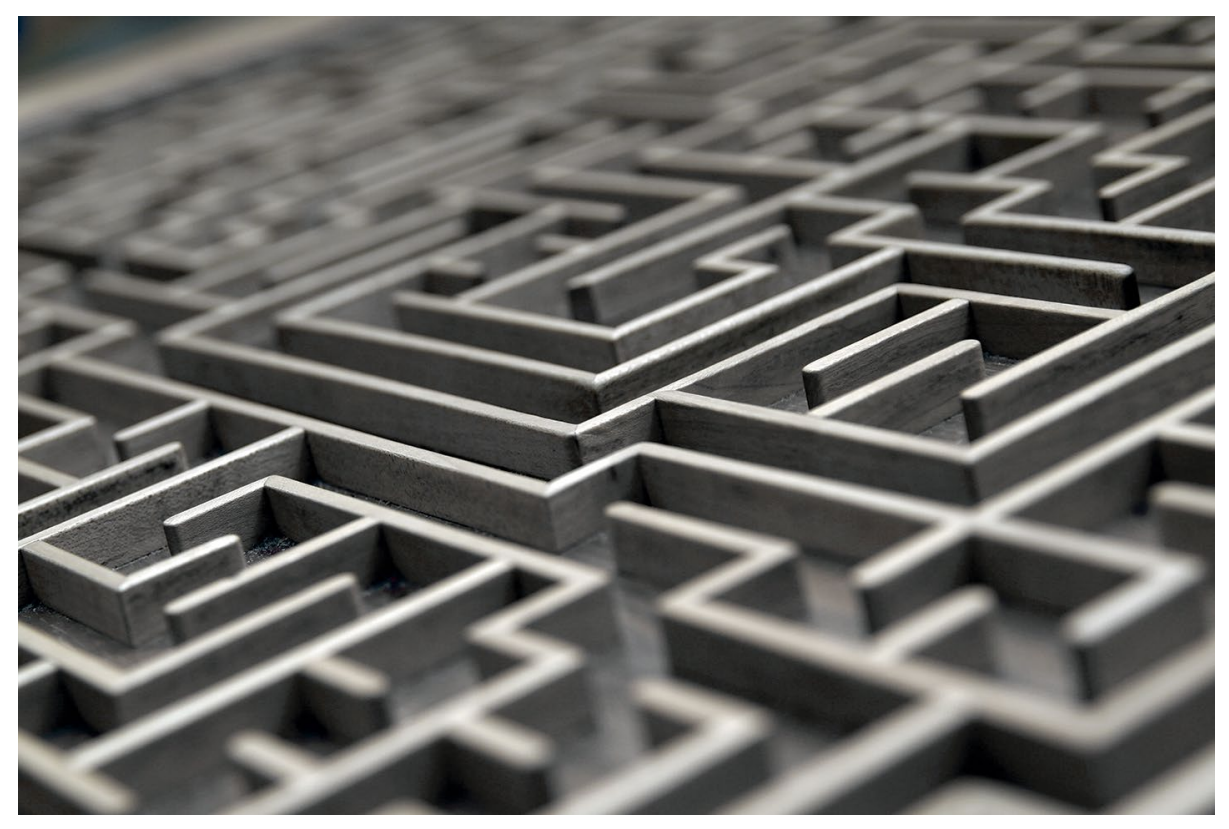

Scientists are exploring many avenues to find out what underpins long-haul COVID. The diverse, often debilitating sets of symptoms are already affecting many, possibly millions, of people around the world. Credit: TwilightEye/Getty

network of long-COVID specialist clinics, as have academic medical centers in the US and elsewhere.

The NIH has launched a research initiative focusing on the Post-Acute Sequelae of SARS-CoV-2 Infection (PASC), which currently has $\$ 1.15$ billion in funding. A cohort of patients will be recruited and followed, and their bodies and biopsies will be scrutinized using a broad assortment of technologies. The NIH PASC Initiative will assess, among other aspects, how many people develop such symptoms after SARS-CoV-2 infection and will explore their underlying causes. Some research opportunities have been announced as part of the initiative, and more are forthcoming.

Long COVID will take time to figure out: "We are only at the beginning of it," says Nadia Rosenthal, scientific director of The Jackson Laboratory (JAX). Given the urgency, "We are all collaborating like crazy." Even scientists whose work has been far afield from virology are eager to work on acute and long-haul COVID-19. "Our motivation level, collectively, it's extremely high," says Nath. Right at the beginning of the pandemic, many NIH labs-like many labs around the world-pivoted their research to SARS-CoV-2. This needs to continue, says Nath, not only because of this virus but because of the next viruses we are likely to face. And given the virus's effect on many of the body's organs, including the lungs, heart, brain and kidneys, "a multidisciplinary approach makes a lot of sense," says Nath.

\section{Is there a viral reservoir?}

Even months after an infection, mRNA from SARS-CoV-2, as well as viral protein, have been detected in the intestines of infected individuals. Michel Nussenzweig and colleagues at Rockefeller University 


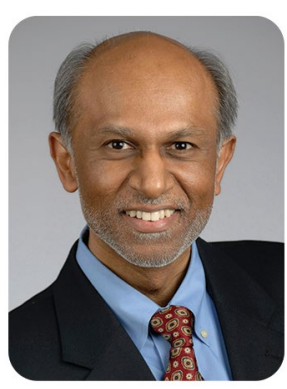

"The virus may be gone, but the music lingers on," says Avi Nath. Perhaps this is due to the immune system or virus remnants. Credit: NIH NINDS

and several other universities have been looking at how antibodies to SARS-CoV-2 evolve and how memory B cells of the immune system remain primed for antibody production in case of reinfection ${ }^{4}$. Four months after onset of COVID-19, immunofluorescence and PCR analysis of intestinal biopsies showed persistence of viral RNA and protein. There is a vestige protein that the immune system is reacting to, says Rosenthal; an antibody "is picking up something," she says. That does not automatically mean this is what is making people ill, "but it could."

Nath says the finding of potential viral reservoirs "to me, is very fascinating." Some viral infections are known to live in reservoirs in the body, but they tend not to induce an inflammatory response. $\mathrm{He}$ thinks back to an Ebola research initiative led by the National Institute of Allergy and Infectious Diseases (NIAID), during which he tended to people in Liberia infected by Ebola virus who had neurological symptoms. Nine months after infection, men still had virus in their seminal plasma.

Susan Weiss at the University of Pennsylvania has long studied coronaviruses, and she wants to learn more about the persistence of SARS-CoV-2 and viral RNA. RNA does not integrate into the host genome, she says, but when a mouse is infected with murine coronavirus, viral RNA can persist in its central nervous system (CNS) without infectious virus being present. The virus infects the liver and the CNS, but persists just in the CNS. This has puzzled the field "for decades," she says. The RNA can remain for the mouse's whole lifetime, and "this is associated with demyelinating disease," says Weiss. There is "no evidence at all for this in humans so I don't really want to make an analogy-just an interesting fact." Brodin believes that intense study of viral reservoirs, viral persistence and related aspects should be a focus in long COVID and beyond, for example for diseases such as myalgic encephalomyelitis/chronic fatigue syndrome (ME/CFS).

\section{Is it in your head?}

"The virus may be gone, but the music lingers on," says Nath. "What is lingering: is it the immune system that is lingering or is it parts of the virus that are lingering?" He has encountered many people with symptoms after a viral infection that were assumed to be immune-mediated conditions. He recalls one person who was part of the NIH Undiagnosed Diseases Program and was experiencing dementia-like symptoms ${ }^{5}$. The team performed whole-exome sequencing, immunoprofiling; they studied immune cells in his blood. With a phage-display method, they looked in his cerebrospinal fluid and blood for immune cells that target dengue virus and checked for autoantibodies; they assessed metabolites and tested for infectious diseases; they gave him drugs used in multiple sclerosis. The assays were inconclusive, the symptoms didn't cease and the man passed away. The autopsy revealed that in the man's brain, "there was dengue virus all over the place," says Nath. Using immunohistochemistry, in situ hybridization, quantitative PCR and sequencing, the scientists found the virus had persisted in his central nervous system and brain, and it appeared this had led to panencephalitis and progressive dementia.

Nath has spoken to around 200 people with long-haul COVID, and there are plans to bring many people in to the NIH for intensive study. In this long-COVID group, once complications due to conditions such as diabetes are excluded, he sees three ailment categories: exercise intolerance, dysautonomia and 'brain fog'. Many people do not get better on their own, but some do. "There is a little bit of hope in that regard," he says. Exercise intolerance, for example, is a condition that a cardiologist in this group of long-haulers faces. Taking the flight of stairs to her office so exhausts her that she must spend the rest of the day in bed. Even after a complete cardiac workup, "they can't find anything wrong with her," he says.

"Dysautonomia - that some people call POTS syndrome," says Nath, for postural orthostatic tachycardia syndrome, involves a wide variety of symptoms in people with long-haul COVID: their heart races and they get dizzy when they stand too quickly as their blood pressure plummets. One patient, herself a neurologist, must speak with Nath lying down to avoid this blood pressure plunge.

Brain fog in people with long COVID appears to afflict particular areas of the brain, says Nath. They are unable to remember names of people and objects, and they lose a sense of time. They can recount what they had for breakfast but fail to recall if it was today's breakfast or last week's, he says. Some people experience mood changes. One long-hauler suddenly experienced suicidal thoughts, which alarmed him so much that he admitted himself to the hospital and has been treated successfully.

Nath has heard that some people suffering from long COVID report feeling better upon vaccination. "This is known to occur in other diseases," he says. For example, people with ME/CFS will report feeling better after getting a flu vaccine. Although for some the fix is permanent, for most people it's only transient, he says. "But it does tell you about the underlying pathophysiology." If long-haul COVID is an inflammation syndrome, as many say, then the activation of the immune system by a vaccine might not necessarily make people feel better. He has no answers but some hypotheses that draw on his background as a virologist and build on what's known about how viruses can change their mode of replication.

Months, even years, after recovering from measles, some children develop a deadly condition called subacute sclerosing panencephalitis (SSPE). Hunting for virus in the sick child's body yields no findings. At autopsy, "you look at the brain, it's loaded with the virus," says Nath. What has taken place is that measles virus remains in the brain and it has mutated to the point at which it no longer forms a complete viral particle. It replicates only in a restricted form: it will form some RNA, some proteins and "it even has the ability to go from cell to cell," he says. The changes allow the restricted virus in one neuron to fuse itself, along with its RNA and protein, with the cell membrane of a neighboring neuron. It keeps moving, infecting a succession of neurons.

The viral genome in these instances reveal two mutation types, he says. One affects the matrix protein, and it's a change

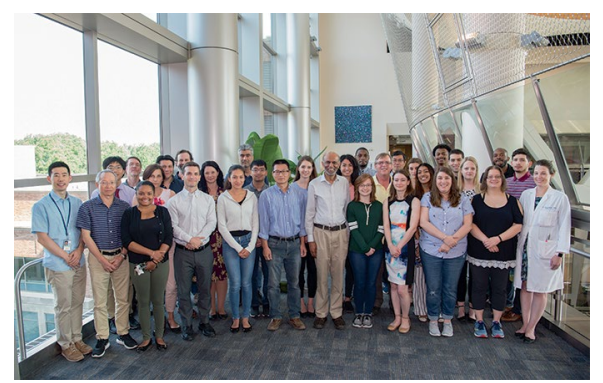

The NIH has launched a $\$ 1.15$ billion initiative focusing on long COVID. In samples from people with long COVID, the NINDS lab of Avi Nath is looking for viral remnants or viral signatures. Credit: NIH NINDS 
that prevents the virus from forming a complete particle. The other mutation occurs in the fusion protein and makes the virus more fusogenic. This restricted replication, says Nath, must offer the virus evolutionary advantages, such as an opportunity to recombine with other viruses to form new ones.

As part of his upcoming work on long-haul COVID-as an intramural researcher, he, too, applies for grantshe hopes to check for viral remnants or viral signatures and check for evidence of restricted viral replication in people. That will require immunological profiling of the long-haul COVID cohort now being set up. All the 'toys' at the NIH intramural program will be put to work, he says. They will use, for example, the 7-tesla MRI for brain imaging to see if there are remnants of viral pathology in the brain that otherwise can only be seen at autopsy. "That's our goal," he says. His lab will also collaborate with David Goldstein's lab at the NINDS for neurotransmitter analysis.

The PASC initiative will involve projects that follow a cohort of people suffering from long-haul COVID. They will be admitted to the NIH's hospital for many types of tests. "We'll study them in great depth," says Nath. Beyond the aforementioned MRI scans, there will be single-cell sequencing of cells in the spinal fluid, an immunological workup of their blood, sleep studies, cognitive tests and detailed autonomic testing. PASC also involves an autopsy cohort and detailed analysis of tissue from individuals who suffered from long COVID_including genomic and transcriptomic analysis and various types of imaging, such as electron microscopy.

Investigators within and outside the $\mathrm{NIH}$ will probe long-haulers in PASC's SARS-CoV-2 Recovery cohort for symptoms and characterize their biopsies. Scientists will also draw on data from NIH initiatives such as the Community Engagement Alliance Against COVID-19 Disparities. There will be a PASC investigator consortium and resources for sharing anonymized biospecimens and data with the wider research community.

\section{Is it in your genes?}

As with all biological and medical problems, says Casanova, in this case what's best suited to parsing human phenotypes seen in COVID-19 is genetic analysis. In a large-scale, international COVID project, Casanova and his colleagues showed that type I interferon plays an important role in COVID-19 and that mutations affecting type I interferons can make it more likely

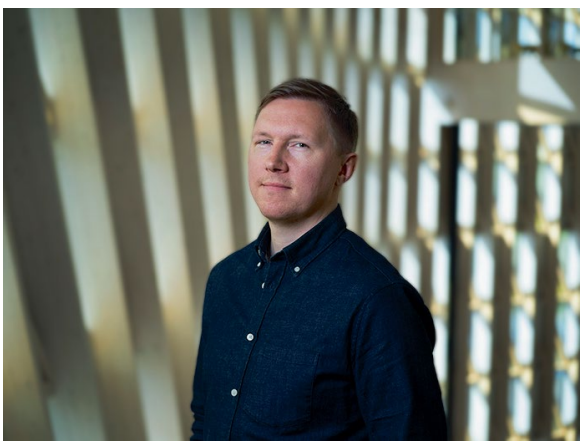

In studying conditions such as long COVID, it matters to apply systems immunology: a focus on the interactions and interplay among immune system components, says Petter Brodin. Credit: Karolinska Institute

that the disease will take a severe course in some people. Could that offer indications about long COVID ${ }^{6,7}$ ? "Yes," he says. "We are on it."

Labs might only use immunoprofiling, but he sees drawbacks in doing so. "The problem with the immunological approach is that you don't know what's the egg and the chicken," says Casanova. "Are the anomalies seen causes or consequences of long COVID?" Genetics has helped scientists understand susceptibility to and severity of pneumonia and a condition called multisystem inflammatory syndrome in children (MIS-C), and "it may work for long COVID," he says. What makes genetics-based approaches strong is that they take researchers "to the root cause," he says.

Brodin agrees on the importance of genetics. Acting atop this layer of genetics that has been shaped by evolution are the influences from the environment. These layers "together determine outcome of COVID and any other infection," says Brodin. Along with others, Casanova and Brodin have organized the Human COVID Genetic Effort, an international consortium devoted to finding the human genetic and immunological basis for the diversity of SARS-CoV-2 infection's clinical course.

Brodin leads the consortium's long-COVID group. The plan is to share ideas and samples and perform analyses of patients who develop long COVID, says Brodin. "We are happy to welcome new members and both ideas and suggestions, also." The focus is on patients with milder forms of acute COVID-19 who are 12 weeks or more beyond their acute bout but still have long, persistent fevers, dysautonomia or other forms of "clearly abnormal physiological presentations," he says. "We need to know if there is virus left in the body, but this is very hard to measure in humans," he says. They plan to perform deep sequencing of genomes, use imaging to hunt for viral particles in tissue specimens and assess autonomic dysregulation. "Why some are affected, and most not, is unknown and a big focus for us," he says. Another research prong relates to immune dysregulation and autoimmunity. It will involve profiling autoantibodies and applying systems immunology, which he describes as a focus on the interactions and interplay among immune system components. This approach is "in contrast to taking the system apart to study its components in isolation." It's a mindset he hopes can take hold, given that "the immune system is an integrated system of cells and we need to think of it as such."

Beyond COVID-19, Brodin focuses on children and their immune systems. As a pediatrician, "I am used to this developmental perspective and thinking of things in that regard." Many different techniques can used for this sort of inquiry, and they all have their unique strengths and weaknesses. "Single-cell resolution is critical, but other than that almost anything goes," he says.

In one of his lab's projects, the team performed systems-level immunomonitoring of people with COVID-19 and tracked them from their acute to their recovery phase ${ }^{8}$. During the acute phase of the disease, the immune cell composition in the body changes dramatically. For example, neutrophils are over-represented compared with lymphocytes. This reverses during recovery. From the acute phase to recovery, basophils and eosinophils increase. These cell types changed the most during acute disease which, the team believes, points to their importance in antiviral defense and immunopathology. Because the disease responses change so much over time, labs are obtaining different findings. "So much depends on timing and when samples are collected because the disease is so dynamic," says Brodin. It's why the team followed people over time. This longitudinal

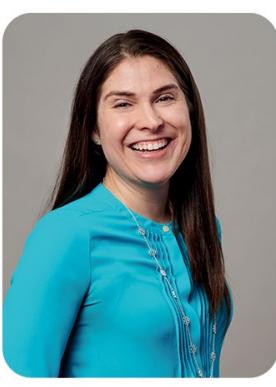

Among the mouse models in development are animals that develop mild symptoms of COVID-19, says Terina Martinez. Credit: Taconic Biosciences 
approach will be needed with long COVID, too.

In their study, Brodin's group used mass cytometry for immunophenotyping and automated cell classification. They homed in on the big changes in eosinophils and used an approach called partition-based graph abstraction to reconstruct single-cell phenotypic changes in eosinophils in the blood. Eosinophils have a previously underappreciated role in the acute response to SARS-CoV-2.

\section{How to model long COVID?}

"We've seen a lot of pivots," says Terina Martinez, a field application scientist at Taconic Biosciences, which develops and sells mouse models. When SARS-CoV-2 emerged, labs that might have been working on, say, IL- 6 or TNF- $\alpha$ and chemotherapy-resistant tumors urgently switched to focus on COVID on accelerated timelines. Taconic helps labs connect with locations offering access to high-security labs so researchers can test hypotheses about SARS-CoV-2. They also help with administrative hurdles. "If it takes you 10 months to sign an MTA, that really slows down the rate of science," she says, referring to material transfer agreements. The first viral strains of SARS-CoV-2 did not infect mice, because the animals have a slightly different form of angiotensin-converting enzyme 2 (ACE2) receptor, which the virus uses to enter human cells. But at the start of the pandemic, Taconic had a mouse model at the ready that had been developed at the University of Texas Medical Branch in Galveston as part of research into SARS-CoV-19. The models are transgenic mice that express human ACE2 (hACE2) that is placed under the regulation of a global promoter. With this preclinical model, the difficulty is that the mice get acutely ill very quickly, says Martinez. This might be connected to how densely the receptor is distributed in the animal, which was generated using random transgenesis methods. Given the many open questions, she says, the company has been working to generate other models, using "the entire modern genetic toolbox" with its different types of gene editing and gene targeting, to create a better tool to address long COVID.

They also plan to develop models of mild COVID-19 symptoms, which might be achieved with animals with different hACE2 expression levels. Also in the pipeline are gene-edited mouse models in which the mouse ACE2 is entirely knocked out and replaced by the hACE2 gene, she says. Each model has pros and cons, and each will be useful for different kinds of experimental questions. Model development is iterative

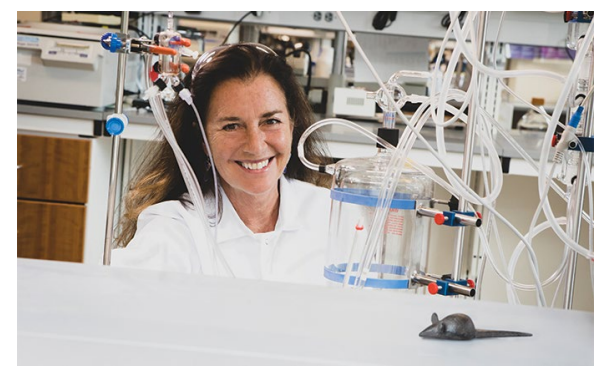

SARS-CoV-2 is "beautiful in a terrifying way, because of the extraordinary intelligence of this evolutionarily adjusted semi-living thing," says Nadia Rosenthal, scientific director of The Jackson Laboratory. Credit: JAX

work, Martinez says: sometimes what is planned on paper, and what people think occurs genetically, "may not play out that way with biology."

The diversity of human response to COVID-19 and the many symptoms of long COVID puzzle and intrigue Rosenthal. Patients with long COVID tend to be younger than those with acute symptoms. The virus "incites riot in the immune system," so it's tempting to think this continues in long COVID. Yet "it kind of doesn't fit the data," she says, given that there is not much of a causal relationship between the initial response to infection and what occurs later. The reactions to the virus are very diverse, and it's true that "you can't use one inbred mouse to represent the entire human race," she says.

People differ genetically, and their immune systems are shaped by their genetics and environmental influences. These differences could indicate who is more susceptible to long COVID and help with teasing out the underpinnings of different long-haul symptoms. Most of the current animal models of COVID-19 have an acute course, given how the virus invades cells through hACE2. "It's beautiful in a terrifying way, because of the extraordinary intelligence of this evolutionarily adjusted semi-living thing," says Rosenthal.

When the pandemic emerged, JAX sent labs working on antivirals and vaccines a mouse developed over a decade ago in the lab of immunologist Stan Perlman at the University of Iowa and his then postdoctoral fellow Paul McCray, who now has his own lab at the university. The mouse was engineered to express ACE2 on epithelia, with its expression under the control of a mouse epithelial promoter gene. The mouse has been used in many studies, including those that led to the vaccines, but its expression is high and mislocated, says Rosenthal.
"That's our theory about why this mouse is not the perfect model."

She had reached out to colleagues at the NIH NIAID Rocky Mountain Laboratories in Montana. "I've got this crazy idea," she recalls telling them. "I want to put that particular transgenic construct onto many different backgrounds." She wanted to cross the mouse with some of the JAX strains to explore shifting the one phenotype to reveal more of the diversity of symptoms in people. The COVID Diversity Mouse Initiative was born.

Sonja Best and Shelly Robertson at Rocky Mountain Labs agreed, and Rosenthal got a green light from Steven Holland, who directs intramural research at the NIAID. Pre-COVID, Rosenthal and Holland had discussed modeling genetic diversity in infectious disease in relation to Zika and Ebola. When COVID-19 hit, she called him to say: "It's show time," to which he responded, says Rosenthal: "You bet." In a few weeks, the project was up and running.

Mice with diverse genetic backgrounds have since been bred from eight inbred mouse strains, and her Rocky Mountain Labs colleagues tell her they are surprised by the animals' behavioral diversity and their diversity of responses to SARS-COV-2. In some strains, only the males get sick; in another only the females do. After infection with SARS-CoV-2 in one mouse strain, "one guy just walked away, gained weight," says Rosenthal, not unlike an asymptomatic human. Some animals dropped weight but bounced back, whereas others dropped weight and died. The scientists have only just started analyzing the data.

Some people with long COVID get bad lung infections, develop thrombosis or lose their senses of taste and smell. Perhaps, she says, the animal's genetics can indicate if it's likely to develop lesions in the hindbrain, which is right next to the olfactory bulb. Such changes could explain why long COVID affects sense of smell and taste. "I don't know," she says. "We're still doing all the histology on these animals."

Brain fog and heart palpitations are occurring in unpredictable ways in people with long COVID. One of these latest JAX animals is showing microthrombi in the heart. "The clotting cascade is a very likely target for this virus," she says. The team is looking mainly at lung and brain tissue and will expand to other tissues later. "We're also doing a huge amount of transcriptomics," she says. That will take a while, but it will yield information about expression quantitative trait loci (eQTLs), which are genetic patterns used to characterize networks of genes. The team will carry out 


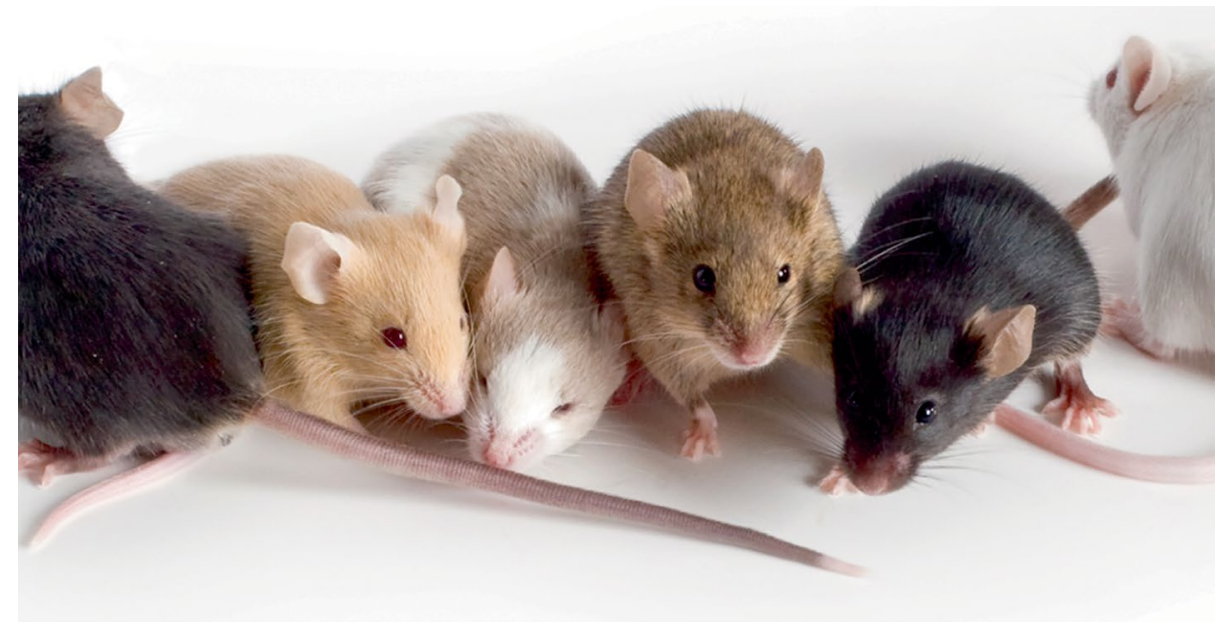

The Jackson Laboratory is working to diversify current mouse models to make them better represent variability between people. These mice could help to model the puzzling diversity of long-COVID symptoms. Credit: JAX

eQTL analysis on a number of different tissue types.

The team is now also developing a series of mice with the hACE2 receptor and human genetic regulatory machinery. Because modeling long COVID means assessing mice for long periods, Rosenthal and her team are developing an aging program to do so. "I can't wait to get those mice aged out and see what happens to them," she says.

Labs need large numbers of mice to lend experiments the statistical power to draw biomedical conclusions. "I just wish I could put the clock forward nine months and just see what happens," she says. She believes insights could emerge that enable genetics-based predictions of how a COVID-19 infection will play out in an individual and "maybe even predict that you are not susceptible to long COVID," she says.

In March 2020, Rosenthal convened an informal JAX group open to all staff to brainstorm and talk through experiments to develop better mouse models for COVID-19. More and more people from all ranks kept joining the weekly meeting she now calls the COVID Mouser group. Ideas are discussed on topics such as how genes might affect one another to lead to a plethora of symptoms. Then other members she calls "wizards" develop practical experimental designs to test these hypotheses. "We have a panoply of new models to test," she says.

They and other scientists have been exploring how to adapt the virus, too. Ralph Baric at the University of North Carolina has been passaging the virus through the mouse system to nudge it toward an artificial evolutionary shift, in which the mouse's physiology forces the virus to mutate. The JAX team is testing this "mouse-adapted virus" on several of the genetically diverse mouse strains. According to quite preliminary data from other labs, says Rosenthal, mice without the hACE2 receptor become infected with the SARS-CoV-2 P.1 variant that was first detected in Brazil and the B.1.351 variant that emerged in South Africa.

If mice with the same genetic background develop the same symptoms repeatedly, "that tells us that the genetics is there to unravel," says Rosenthal. A chunk of DNA with perhaps hundreds or thousands of genes might be tracking with a response. Not all of the symptoms of long COVID will have direct parallels in mice, "but I think we're going to get damn close with some of these animals. And we've just begun."

\section{How to join in?}

It's not a must for everyone to study COVID-19, says Nath. But postdoctoral fellows and graduate students in relevant fields who are not working on COVID should follow the literature. So much technology is going to be thrown at COVID and long COVID, he says, and sophisticated science will result. "New technology will develop because of it," he says. "And so you will learn a lot that you can apply to other diseases."

Other diseases have not disappeared, however, and people are still also dying from cancer or Alzheimer's or Parkinson's diseases. "I wouldn't recommend that everybody just stop what they're doing and start doing COVID," he says. If people follow their passion and expertise, and follow the literature, they can learn from it and apply it. And if they develop tools that can apply to COVID and help others, he says, "share it with them."

\section{Vivien Marx ${ }^{凶}$ \\ Nature Methods. \\ $\bigotimes_{e-m a i l: v . m a r x @ u s . n a t u r e . c o m}$}

Published online: 28 April 2021

https://doi.org/10.1038/s41592-021-01145-Z

References

1. Carvalho, T., Krammer, F. \& Iwasaki, A. Nat. Rev. Immunol. https://doi.org/10.1038/s41577-021-00522-1 (2021).

2. Nalbandian, A. et al. Nat. Med. https://doi.org/10.1038/s41591021-01283-z (2021).

3. Bhadelia, N. et al. Preprint at medRxiv https://doi.org/10.1101/ 2021.01.21.21249176 (2021).

4. Gaebler, C. et al. Nature 591, 639-644 (2021).

5. Johnson, T. et al. Ann. Neurol. 86, 695-703 (2019).

6. Zhang, Q. et al. Science 370, eabd4570 (2020).

7. Bastard, P. et al. Science 370, eabd4585 (2020).

8. Rodriguez, L. et al. Cell Rep. Med. 1, 100078 (2020).

9. Tseng, C.-T. K. et al. J. Virol. 81, 1162-1173 (2007). 